\title{
TIME TO CHOOSE BETWEEN SCIENTIFIC AND ADMINISTRATIVE APPROACH TO RELIABILITY
}

\author{
Dr Jezdimir Knežević * \\ MIRCE Akademy, Woodbury Park, Exeter, United Kingdom
}

\begin{abstract}
"If you watch a glacier from a distance, and see the big rocks fallings into the sea, and the way the ice moves, and so forth, it is not really essential to remember that it is made out of little hexagonal ice crystals. Yet if understood well enough the motion of the glacier is in fact a consequence of the character of the hexagonal ice crystals. But it takes quite a while to understand all the behaviour of the glacier (in fact nobody knows enough about ice yet, no matter how much they've studied crystal). However, the hope is that if we do understand the ice crystal we shall ultimately understand the glacier."
\end{abstract}

R. Feynman, "The Character of Physical Law"

\section{INTRODUCTION}

Reliability Theory, since it's beginning in 1950's, has been based on mathematical theorem rather then on scientific theories. Massive attempts where made to further applications of the existing mathematical and statistical methods and analysis without attempts for understanding "failure mechanics". Then, in 1980s, practicing reliability engineers and analysts, who have neither ability to understand the mathematics, turned to what they have had, which is enormous practical experience of the observed failure modes of existing systems. Thus, a large number of "practical reliability methods" have been developed and used, all of which were based on the Failure Mode, Effect and Criticality Analysis, FMECA, but still without any attempt to understand and address physical mechanisms that generate failures. Consequently, during the last 50 years the Reliability Theory made very little progress, a part from a few exceptions (one should put some references here), in the direction of becoming the science, in terms of making accurate predictions that could be confirmed with practical observations. The reason is very simple; neither statistics, which does not study causes of statistical behaviour, nor engineers whose "applied methods" were focused on meeting contractual and legal requirements, by doing FMECA to "prove" Mean Time Between Failures, MTBF, were able to provide a fertile ground for the development of reliability.
To illustrate the above statement the fundamental expression for reliability will be used. It is generally accepted that reliability is the probability that a system will operate without failure during a stated period of time, which is mathematically represented by the following expression:

$$
R(t)=P(T T F>t)
$$

where: TTF is a random variable known as the Time To Failure and $\mathrm{R}(\mathrm{t})$ is the reliability function.

However, today there are two distinguished approaches to calculation of the probability defined by the above equation. They are:

Approach 1, where calculation of the probability of a successful operation with internal of time from 0 to $t$ is based on the following expression:

$$
P(T T F>t)=\prod_{i=1}^{n f m} P\left(T T F_{j}>t\right)
$$

where $n f m$ is a total number of competing failure mechanisms that can generate a failure event. It is necessary to stress that a probability distributions that define individual failure mechanisms are exclusively determined by the physical processes that generate them, like fracture, single event upset, electrostatic discharge, fatigue, creep, wear, radiation, hot electron, embrittlement, depolymerisation, charge trapping in oxides, glass transition and many others.

Approach 2, well established within western defence aerospace, oil and other industries, for all reliability predictions, risk and safety as 
sessments, conformances, contracting and similar activities, where the probability of operation without failure during a given interval of time $t$ is defined by the following expression:

$$
P(T T F>t)=e^{\left[-\left[\sum_{i=1}^{n f m} \lambda_{i}\right) t\right]}=e^{\left[-\left(\frac{t}{M T B F}\right)\right]} \text { where MTBF }=\frac{1}{\sum_{i=1}^{\text {vffut }} \lambda_{i}}
$$

where: $\lambda_{i}$ is the failure rate of each failure mechanisms that can generate a failure even.

Both expressions for reliability function clearly demonstrate that the system reliability follows the laws of probability. However, the expression 2 allows the probability laws to be driven by physical processes and mechanisms that take place in the system or result from the interaction of a system with natural and human environment, whereas the expression 3 has one, and only one, predetermined future, irrespective of physical properties of systems, their operational conditions, maintenance policies and support strategies. In fact the second approach completely ignores existence of corrosion, fatigue, creep and many others, scientifically observed and well understood mechanisms, which have time-dependent failure mechanisms. To make the distinction between these two approaches to reliability the former will be called the scientific approach and the latter the administrative approach.

Consequently, the main objective of this paper is to argue that the scientific approach to reliability is the only way forward for all members of the reliability community who wish to make accurate predictions that will be confirmed during the operational processes of the future systems. Only then, accurate and meaningful reliability predictions become possible, which is imperative for the development of Risk-Based Technology and its successful applications.

\section{SCIENTIFIC APPROACH TO RELIABILITY}

Mathematically, reliability is defined as a probability that a system will maintain a required function during a stated period of time (see equations 1, 2 and 3). However, as a probability cannot be seen or measured directly, engineers and managers, have fundamental difficulty in understanding and interpreting statistical and probability functions associated with their systems. This is because physical characteristics of a system like the weight, temperature, volume and similar have a clear and measurable meaning. Howev$\mathrm{er}$, the concepts of probability, and hence reliability, is an abstract property of a system that obtains a physical meaning only when behaviour of a large sample of systems is considered. Hence, understanding of reliability is reduced to the scientific observation and analysis of system failures, which are observable and measurable physical phenomena.

According to the Mirce Mechanics, system failures are events that cause transition of a system from positive to negative functionability state [1] due to some of the following reasons, or combinations of them:

a) Built-in design errors (incorrect selection of materials, stresses shapes, etc)

b) Production problems (human errors, material and process deficiencies)

c) Irreversible changes in the condition of components with time due to wear, fatigue, creep, corrosion, and similar degradation processes

d) Imposition of external overstress mechanisms resulting from collisions, harsh landings, extreme weather conditions, etc

e) Human errors in execution of maintenance tasks

f) Human errors in execution of in-service support tasks

At the MIRCE Akademy a large number of failure events and associated phenomena have been observed and analysed to understand the physical mechanisms that generate occurrences of failures.

Consequently, systematic studies are applied to understand phenomena that cause thermal aging, thermal buckling, photo-chemical degradation, reduction in dielectric strength, evaporation, metal fatigue, actinic degradation, photo-oxidation, swelling/ shrinking, degradation of optical qualities, fogging, photochemical decomposition of paint, blistering, warping, thermal stress, breakdown of lubrication film, increased structural loads, shift in the centre of gravity, jammed control surfaces, attenuation of energy, clutter echoes, blocking of air intakes, decreased lift and increased drag, unequal loading, removal of coating protection, pitting, roughening of the surface, acid reactions, leakage currents, promotion of mould growth, reduction of heat transfer, caking and drying, premature cracking, hot spots creation, erosion, bleaching preservatives, 
abrasive wear, corrosion, alkaline reactions and similar.

For years, research studies, international conferences, summer schools and other events have been organised in order to understand just a physical scale at which failure phenomena should be studied and understood. In order to understand the motion of failure events it is necessary to understand the physical mechanisms that cause the motion. That represented a real challenge, as the answers to the question "what are physical and chemical processes that lead to the occurrence of failure events" have to be provided. Without accurate answers to those questions the prediction of their future occurrences is not possible, and without ability to predict the future, the use of the word science becomes inappropriate.

After a numerous discussions, studies and trials, it has been concluded that any serious studies in this direction, from Mirce Mechanics point of view, have to be based between the following two boundaries:

- the "bottom end" of the physical world, which is at the level of the atoms and molecules that exists in the region of 10-10 of a metre [03],

- the "top end" of the physical world, which is at the level of the solar system that stretches in the physical scale around $10+10$ of a metre. [04]

This range is the minimum sufficient "physical scale" which enables scientific understanding of relationships between system operational processes and system operational events. In other words, this is the physical range within which, the system operational processes mentioned above (fatigue, the wind direction change, suncups formation on the blue ice runway, bird strike, perished rubber, carburettor icing) take place and as such they could be understood and predicted.

\section{THE BOTTOM END: ATOMIC SYSTEM}

All matter in the Universe is made of elementary building blocks called atoms. Complex interactions between atoms govern existence of larger building blocks. [2] For example two or more atoms form molecules, ranging from simple oxygen molecules to large polymers and other macromolecules. Besides this way of building the matter, atoms can arrange in periodic structures called crystals. Examples of crystals are numerous, from the rock salt (crystal of $\mathrm{Na}$ and $\mathrm{Cl}$ ), over diamond (made of $\mathrm{C}$ atoms) and crystal of
Iron to recently synthesized crystals in the field of Nanotechnology, to mention just nanotubes and graphene - the miracle materials with large promise for the future applications. While the average size of atoms is $10-10 \mathrm{~m}$ crystals can grow to macroscopic dimensions of the order of a meter, making objects like airplane wings, car bodies etc. The very atomistic nature of these objects governs their mechanical, electronic, thermal and other physical properties, which are of interest for Mirce Mechanics. Additionally material defects, fatigue and other features, which can in the final instance, lead to the failure of material and finally a cancellation of flight or even a disaster, are originated at the atomic level. Quantum mechanics, a physical theory developed in 1920s, in exact way describes the matter at the atomic scale. This theory has the power to predict the evolution of material under stress, corrosion or other environmental influences, which complements Mirce Mechanics, giving meaningful values to the missing parameters of the theory.

\section{THE TOP END: SOLAR SYSTEM}

The Solar System may seem enormous, looking from the human perspective, but it is only a very small corner of the Universe. However, the entire solar system contains only eight planets that move in elliptic paths around the Sun. All of them are lit by the Sun and do not produce their own light. The distance between the Earth and the Sun is 150 million kilometres; hence the number for the top end of 1010. Thanks to its thermonuclear reactions which last for 5 billion years, the Sun irradiates enormous energy each second in the form of electromagnetic and other radiations, out of which only $\sim 1 / 109$ fraction reach the Earth. Owing to them rivers flow, winds blow, forest rustle and the human race flourish.) About a half of that energy $(0.8 \times 1017$ watts) reaches the terrestrial surface, which is $5 \times 1014$ square metres, making the average power of the solar radiation at ground level is 160 watts $/ \mathrm{m} 2$. The $99.9 \%$ of it is absorbed by the soil, and goes into the evaporation of water, causing winds, thunderstorms, and all that we loosely call weather. Thus, only 0.1 per cent of the radiant energy of the Sun (around 1014 watts) is captured by plants through photosynthesis of organic substances from carbon dioxide and water. This energy supports all the living things on Earth, from bacteria to animals and human. 
From system reliability point of view, the solar system is significant in the respect to the "making" of the weather, which is the day-to-day condition of the atmosphere. It is one of the main drivers of system reliability, as it is "responsible" for the:

- temperature and pressure of the air,

- wind speeds and directions,

- moisture in the air, precipitated as rain, snow, hail, sleet, dew or frost.

All air contains moisture in the form of water vapour, which is water in gaseous form. As warm air can hold more water vapour than cold air, when it is cooled its capacity to hold water vapour decreases, and finally the air is completely saturated, having a relative humidity of 100 per cent, known as dew point. Further cooling beyond dew point leads to water vapour condensing around nuclei, such as specks of dust or salt, to form water droplets or, in cold air, minute ice crystals. Large quantities of condensed water vapour form clouds, by which water is continually conveyed from the oceans to the land, where it is released from the air as precipitation. This provides the land with the fresh water needed by animal and plant life. Finally, the water completes the cycle by returning to the oceans.

\section{AN EXAMPLE: IMPACT OF COSMIC RAYS ON AVIONICS RELIABILITY}

In order to illustrate the necessity for the physical scale of studies of reliability phenomena proposed in this paper to be from 10-10 to $10+10$ of a metre, the impact of cosmic rays on reliability of avionics will be presented here. It has been concern for avionics, since the late 1980's when the primary radiation phenomenon, which had previously been observed in orbiting satellites only, also began to appear in aircraft electronic systems (Put some references here). The interaction of this radiation with avionics can result in occurrence of Single Event Effect, SEE, which can be manifested as a transient 'soft error' effect such as a bit flip in memory or a voltage transient in logic. Alternatively, a 'hard error' can be induced resulting in permanent damage such as the burn out of a transistor. Due to the rapid advances in electronics technology and the unrelenting demand for increased avionics functionality in the competitive commercial aircraft industry, the complexity of avionics systems has risen exponentially. If device memory cells used for flight safety or mission critical functions are affected the concern is that the loss of key system functionality due to corrupted data could cause a flight safety or mission critical failure. Baumann in [3] stated that: "Left unchallenged, SEEs have the potential for inducing the highest failure rate of all other reliability mechanisms combined".

Advanced microprocessor and memory semiconductor devices used in modern avionics exhibit an increased susceptibility to SEEs caused by ionising radiation from the following two main sources:

- Cosmic rays from space (10+10 of a metre and beyond) that are individual energetic particles that originate from a variety of energetic sources ranging from our Sun to supernovas and other phenomena in distant galaxies all the way out to the edge of the visible universe. Although the term cosmic ray is commonly used, this term is misleading because no cohesive ray actually exists. The majority of cosmic rays consist of the nuclei of atoms (atoms stripped of their outer electrons) ranging from the lightest to the heaviest chemical elements. In terms of composition about $90 \%$ of the nuclei are hydrogen, therefore just single protons, $9 \%$ are helium, alpha particles with the remaining $1 \%$ a mix of heavier element nuclei, high energy electrons, positrons and other subatomic particles. Within the atmosphere the three most important parameters used to define the variability of the particle flux at a specific location are: altitude, latitude and energy. Within the field of cosmic ray physics altitude is expressed in terms of atmospheric depth, which is the mass thickness per unit of area in the Earth's atmosphere. Cosmic rays can be broadly divided into two main categories, primary cosmic rays and secondary cosmic rays. Primary cosmic rays are particles accelerated at astrophysical sources and generally do not penetrate the Earth's atmosphere. Secondary cosmic rays are created when primary cosmic rays collide with oxygen and nitrogen nuclei in the atmosphere and break into lighter nuclei in a process known as cosmic ray spallation.

- Alpha particles from radioactive impurities in the materials of which device are made (10-10 of a metre and below). They are doubly ionised helium atom consisting of two 
neutrons and two protons that can also be described as a helium atom that has been stripped of its electrons. When an alpha particle travels through a material it will lose kinetic energy primarily through interactions with the materials electrons, leaving a trail of atoms with "kicked out" orbital valence electrons. This process is called ionisation and it can be described as the physical mechanism that converts an atom or molecule, into a positively or negatively charged state by either adding or removing charged particles. The resulting atom is then referred to as an ion, or more specifically a cation if positively charged or an anion if negatively charged. The issue of alpha particle generating source contaminates first arose in the late 1970s when Intel discovered high soft error rates in new DRAMs when the integration density increased from $16 \mathrm{~K}$ to $64 \mathrm{~K}$. The problem was traced to a semi-conductor packaging plant that had just been built downstream from an abandoned uranium mine. The ceramic packages were being contaminated by radioactive contaminants in the water. Low energy alpha particles are emitted from the decay of trace radioactive materials in semi-conductor device and packing materials.

The relationship between the radiation particles and the failure mechanisms of the single events upsets is shown in the Table below [04]:

Table 1: Summary of Failure Mechanisms

\begin{tabular}{|c||c|c|c|}
\hline Radiation Type & Radiation Source & $\begin{array}{c}\text { Method of Charge } \\
\text { Deposition }\end{array}$ & $\begin{array}{c}\text { Failure } \\
\text { Mechanism }\end{array}$ \\
\hline Thermal neutrons & $\begin{array}{c}\text { Secondary cosmic } \\
\text { ray neutrons }\end{array}$ & Indirect lonisation & $\begin{array}{c}\text { Interaction between thermal } \\
\text { neutrons and materials containing } \\
\text { the Boron-10 isotope creates } \\
\text { secondary ionising particles. }\end{array}$ \\
\hline \hline $\begin{array}{c}\text { Low energy alpha } \\
\text { particles }\end{array}$ & $\begin{array}{c}\text { Radioactive decay } \\
\text { of uranium and } \\
\text { thorium impurities } \\
\text { located within the } \\
\text { device materials. }\end{array}$ & Direct lonisation & $\begin{array}{c}4 \text { to 9 MeV alpha particle, creating an } \\
\text { electron hole funnel. }\end{array}$ \\
\hline $\begin{array}{c}\text { High energy } \\
\text { neutrons }\end{array}$ & $\begin{array}{c}\text { Secondary cosmic } \\
\text { ray neutrons }\end{array}$ & Indirect lonisation & $\begin{array}{r}\text { High energy neutron collisions with } \\
\text { silicon nuclei. }\end{array}$ \\
\hline
\end{tabular}

As the reliance on avionics systems within aircraft increases so do concerns regarding the reliability of these systems, particularly for those systems, which are considered safety critical. Hence, to take the appropriate mitigating actions and enable decisions to be made at the design stage a method need to be devised that will facilitate the calculation of soft errors rates due not only to quiescent conditions, but also to take into account more exceptional solar influenced events.

The research currently undertaken within the MIRCE Akademy has two main objectives:

- the development of an SEE functionability prediction model

- the use of the model to investigate the influence of space weather, flight route and a multitude of other aircraft and system design factors on the resultant shape of the distribution of SEE initiated failure events throught time.
The main areas of research are: the investigation on the influence of the aircraft structure on the internal neutron flux spectra at specific inside locations of the architectures of future commercial aircraft and the evaluation of the methods and techniques used by the electronics industry today to assess their suitability for the inclusion into the SEE functionability prediction model.

A plethora of device and circuit level simulation methods exist together with a range of empirical techniques exist that could be used at various indentures levels. The integration of these methods into an SEE functionability model may lead to an improved understanding of SEE fault propagation mechanisms resulting in a more accurate prediction of failure events at system level. The final goal is the creation of an innovative SEE functionability prediction model that 
will enable the future behaviour of an avionics system to be predicted for a whole host of different external parameters such as the extremes of space weather or different flight routes.

Furthermore the model should allow system designers the flexibility to examine the full range of system design options such as device selection, system configuration and SEE reduction solutions to allow early functionability improving design decisions to be made, with least investment in time and resources.

\section{CONCLUSION AND RECOMMENDATION}

The main objective of this paper was to present the authors approach to Reliability, one that is based on the laws of science. I do not believe in the existence of parallel universes where the laws are either ignored or bent to accommodate administrative or contractual requirements. A prime example of the later is the well accepted model of system reliability that requires the acceptance of "alternative universes" to support the argument that the components and consequently systems possess a constant, time independent, failure rate, as described by the equation 2 . This approach stems from neither science nor observation, but from imaginary steps envisaged in the minds of its proponents who allowed all laws of science to be suspended. However, this view is in direct opposition to the observed functionability phenomena like corrosion, fatigue, creep, wear, quality problems and many other time dependent physical processes that clearly demonstrated that the components/system reliability for a stated period of time could have increasing, constant and decreasing probability of success in respect to the stage of the life of a system, consisting components and maintenance policies applied, as the science based approach caters for through the reliability function defined by the equation 1.

Finally, it is essential to distinguish the scientific approach to the formulation and modelling of the motion of reliability through the life of a system, contained in Mirce Mechanics and presented in this paper, from administrative approach that is based on reliability models of systems that are created to demonstrate the contractual compliance of the legally binding acquisition processes, in western defence and aerospace industries.
As science is the proved model of reality that is confirmed through observation, the summary recommendation of this paper to reliability professionals is to move from the universe in which the laws of science are suspended to the universe that is based on the laws of science in order for their predictions to become future realities.

It is encouraging to know that Rolls Royce reliability department in Darby, England, routinely recognises over 50 different failure mechanisms in reliability modelling of their jet engines.

\section{ACKNOWLEDGMENT}

I wish with this paper to pass my very best wishes to Dr Jovan Todorovic, retired Professor of Mechanical Engineering from Belgrade University for his $80^{\text {th }}$ birthday. Not that he introduced the subject of Reliability to the University of Belgrade in mid 1970s, but he has done it by applying the scientific way, from the beginning, and enthusiastically shared his knowledge with all of those who were able to embrace it. I was one, of many, benefactors of Professor Todorovic's pioneering work in reliability theory.

\section{REFERENCES}

1) Bader, R.F.W., Atoms in Molecules: a Quantum Theory, Oxford University Press, Oxford, UK, 1990.

2) Baumann, R Radiation-induced soft errors in advanced semiconductor technologies, IEEE Transactions on Device and Materials Reliability, Vol 5, No 3, pp. 305-316, Sept. 2005.

3) Knezevic J., Reliability, Maintainability and Supportability - A Probabilistic Approach, with Probchar Software. pp 292, McGraw Hill, UK, 1993.

4) Todorović, J.: Upravljanje održavanjem na bazi rizika, (2009) Journal of Applied Engineering Science (Istraživanja i projektovanja za privredu, no. 1, p. 23-33

5) Zaczyk, I, Analysis of the Influence of Atmospheric Radiation Induced Single Event Effects on Avionics Failures, Master Dissertation, pp 77, MIRCE Akademy, UK, 2009.

Paper sent to revision: 28.08.2012.

Paper ready for publication: 26.09.2012. 


\section{Appendix 1: Mirce Mechanics Concept}

Mirce Mechanics - scientific study of the motion of functionability through the life of a human made and managed system to:

- Experimentally determine the pattern of the motion,

- Scientifically understand mechanisms of the motion,

- Mathematically defined laws of the motion

- Predict the pattern of the motion of a given system.

Functionability, the ability of being functional, is the fundamental property of in-service performance of any system. It is an emergence property of a system, in time domain, resulting from the complex interactions of natural phenomena, such as fatigue, corrosion, creep, wear, humidity, wind, hail, foreign object damage, solar radiation and similar, on one hand and from human actions taken in respect to the type, content and timing of operational, maintenance and support processes, on the other.

To achieve the above objectives Mirce Mechanics concept, principles and methods have evolved from the experimental, theoretical, computational and applied aspects of research, each of which is briefly described below.

Experimental Mirce Mechanics focuses on the determination of the pattern of the motion of functionability through the life of a system resulting from the occurrence of functionability events. Existing experimental and observed data clearly demonstrate that the motion of functionability through life of a large number of "identical" systems deliver a large number of different functionability patterns, while delivering "identical" functionality. Consequently, it is statistical experiment that requires the use of statistical methods to calculate the average pattern and associated measures. However, as statistics does not study the causes of statistical behaviour it is the task of Mirce Mechanics to scientifically understand the mechanisms that cause the motion of functionability in time. Thus, functionability phenomena that cause occurrence of positive and negative functionability events are subjected to the analyses within physical scale between 10-10 metre (for the understanding atomic and molecular phenomena) and 10+10 metre (for the understanding of cosmic and environmental phenomena).
Theoretical Mirce Mechanics focuses on the mathematical definition of the patterns of the motion of functionability through the life of a system. Mathematically formulated law of the motion, in respect through time, which accurately represents the observed patterns, is defined by the expression, named Mirce Functionability Equation, which has been developed by $\mathrm{Dr}$ J. Knezevic at the MIRCE Akademy. It defines, in the probabilistic terms, the expected patterns of functionability trajectory and associated measures for a given system, operational rules and conditions. Although the laws of probability are just as rigorous as other mathematical laws they are not able to predict the motion of functionability through the life of each individual system, they can only predict the probability of each individual system being in a given functionability state at a given instant of time.

Computational Mirce Mechanics focuses on the quantitative evaluation of Mirce Functionability Equation for a given system and given in-service rules and conditions, as the analytical solutions to these equations are too complex to be solved mathematically. Consequently, it is the task of Mirce Mechanics to develop effective computational methods that will enable construction of models that accurately represent the observed reality of system behaviour, rather then to simplify system reality to cope with mathematical limitations. The Monte Carlo method has proved very successful in Quantum Mechanics for finding practical solutions to multi-dimensional integral equations that are of similar nature to those of the Mirce Mechanics. 